\title{
Regeneration gets physical
}

\author{
As the role of biophysical cues in regulating cell behaviour is increasingly understood, more evidence in \\ the field of bioengineering indicates how such signals can affect cells and tissues.
}

Over a century ago, the anatomist Julius Wolff proposed that bone tissue is capable of adapting its microarchitecture through trabecular reorganization in response to mechanical loads, in what is widely known as Wolff's law of bone remodelling ${ }^{1}$ (Fig. 1). Since this realization established the role of biophysical stimuli in modulating tissue development and remodelling, it is now recognized that such cues can have significant implications at the cellular and molecular levels. Indeed, this notion has now grown into a subfield within biological sciences, called mechanobiology, whose concepts have been employed to elucidate fundamental processes in single cells, tissue growth, disease progression, as well as in the development of materials for tissue repair. Previously, we have published articles focused on mechanobiology ${ }^{2}$ and in this issue we highlight how biophysical cues can have significant implications for a range of tissue types in the field of bioengineering.

The success of biomaterials for a range of therapeutic applications has largely been driven by their ability to be tuned with optimal surface chemistry, bioactive ligands, microarchitecture, and mechanical properties depending on the tissue of interest. An overview of how control over biomaterial physical properties can be adopted to divulge previously unappreciated mechanisms of mechanotransduction is provided by Christopher Chen and colleagues in a Commentary on page 1164 . They indicate the advances made in customizable platforms utilized in mechanobiology. Such in vitro platforms have unlocked the potential to investigate precise molecular processes involved in mechanotransduction, which previously relied largely on complex in vivo models with multiple variables. An Article by Daniel Müller and colleagues on page 1262 is focused on characterizing the process of fibroblast adhesion due to varying biophysical cues. They demonstrate that at the onset of adhesion to fibronectin, fibroblasts sense the mechanical load via specific integrins that rapidly signal the engagement of additional integrins to strengthen cell adhesion.

Mesenchymal stem cells and progenitor cells have been widely utilized in regenerative medicine due to their self-renewal capacity and potential to differentiate into distinct cell types for tissue homeostasis. A major challenge in the field of bioengineering is maintaining these intrinsic properties of stem cells and progenitor cells for the repair and regeneration of specific tissues. Sarah Heilshorn and colleagues report on page 1233 how the stiffness and degradability of hydrogels can affect the stemness of progenitor cells in vitro, demonstrating that matrix remodelling in the absence of cytoskeletal tension can be required to facilitate cell-to-cell interaction and maintain neural stemness in a three-dimensional environment.

The role of time-dependent properties of biomaterials such as viscoelasticity on cell response is becoming increasingly important and recent studies have demonstrated that substrate stress relaxation can affect the growth and differentiation of cells. On page 1243, Ovijit Chaudhuri and colleagues investigate how the viscoelasticity of hydrogels affect chondrocyte phenotype in vitro. They show that hydrogels that are capable of rapid dissipation of elastic stress over time in response to applied strain can promote cartilage-like matrix synthesis by cells. On the contrary, hydrogels with high elastic stress resisting displacement with the newly synthesized cartilage matrix are ultimately detrimental to cell phenotype due to restriction in cell and matrix volume expansion.

Changes in matrix mechanical properties have also been associated with pathological states in tissues such as tumours and fibrotic liver $^{3}$. Fibrosis can occur in response to injury resulting in abnormal angiogenesis and scar formation that may ultimately lead to organ failure. While anti-angiogenesis therapies are available, they have not proven successful in some cases of liver fibrosis. Yanan Du and colleagues describe on page 1252 in vitro models that recapitulate the early and late stages of liver fibrosis. These in vitro constructs were used as templates to investigate the response to anti-angiogenesis drugs, indicating that the drugs are effective in early-stage liver fibrosis and this was confirmed with in vivo disease models. In the late stages of the disease, inhibitors of collagen crosslinking are more favourable. Such models constitute a promising tool to elucidate the mechanisms

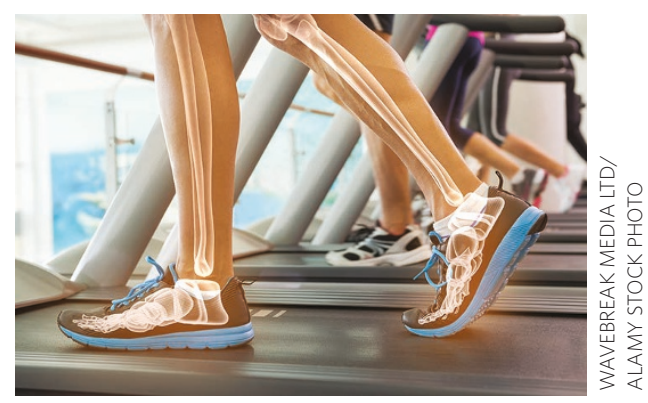

Figure 1| The image shows the skeleton of an individual on a treadmill. Research has indicated the role of weight-bearing activity in influencing bone microarchitecture and mass.

involved in disease progression and unravel the significance of stage-specific intervention in fibrosis.

A major challenge in materials science is the incorporation of knowledge obtained from fundamental research in cell biology into the fabrication of functional and translatable biomaterials. A Perspective by David Mooney and Max Darnell on page 1178 highlights how understanding the intrinsic processes involved in cell and tissue growth could be adopted in the fabrication of optimal biomaterials. By borrowing strategies from bioprocess engineering, the authors describe an approach that involves identification of overarching parameters such as biophysical and biochemical cues that affect cell phenotype and behaviour, subsequently incorporating them in biomaterial development processes to maximize functionality and minimize complexity. As a case study, they demonstrate how this approach may be employed in the development of biomaterials for beta-cell transplantation for application in type I diabetes treatment.

Despite these recent developments, there is certainly a long road ahead to fundamentally understand how biophysical cues modulate cell behaviour. These studies will undoubtedly garner further understanding of mechanisms involved in cell and tissue growth and repair, as well as their response to therapeutic interventions. $\square$

References

1. Wolff, J. The Law of Bone Remodelling (Springer, 1986).

2. Nat. Mater. 13, 531 (2014).

3. Paszek, M. J. et al. Cancer Cell 8, 241-254 (2005). 